\title{
A Survey of Various Protocols Over Vehicle Ad-Hoc Network
}

\author{
Monica Varrmaiya ${ }^{1}$, Sushma Kushwaha ${ }^{2}$, Amit Thakur ${ }^{3}$ \\ M. Tech Scholar, Department of Computer Science Engineering, \\ Swami Vivekanand College of Science \& Tech. Bhopal, India ${ }^{1}$ \\ Assistant Professor, Department of Computer Science Engineering, \\ Swami Vivekanand College of Science \& Tech. Bhopal, India ${ }^{2}$ \\ Associate Professor, Department of Computer Science Engineering, \\ Swami Vivekanand College of Science \& Tech. Bhopal, India ${ }^{3}$
}

\begin{abstract}
Intelligent Transportation Systems (ITS) is a coordinated methodology being created to trade important information to expand the security and proficiency of the road transportation systems. Vehicular Ad hoc Network (VANET), a variation of Mobile Ad Hoc Networks (MANET), is a center component of ITS. IoV offers a couple of advantages, for instance, road prosperity, traffic efficiency, and infotainment by sending best in class traffic information about approaching traffic. Execution of this brilliant ITS for the most part owes to the plan of productive routing protocols in VANETs. The computation of the best course estimates the exhibition of communication while routing protocols deals with communication and routing of the information. Provision of keen communication, requires the examination of routing protocols in VANET. In like manner in this paper, evaluated different sorts of existing routing protocols in VANET, posting their advantages and disadvantages. In conclusion potential inclinations of imminent research connected to VANET routing are discussed.
\end{abstract}

Keywords: Protocol, VANET, ITS, MANET

\section{INTRODUCTION}

An ad hoc routing protocol is a convention, or standard, that controls how hubs choose what direction to course bundles between processing gadgets in a mobile ad hoc network. In ad hoc networks, hubs are inexperienced with the topology of their networks. Instead, they need to find it: ordinarily, another hub reports its quality and tunes in for declarations broadcast by its neighbors.

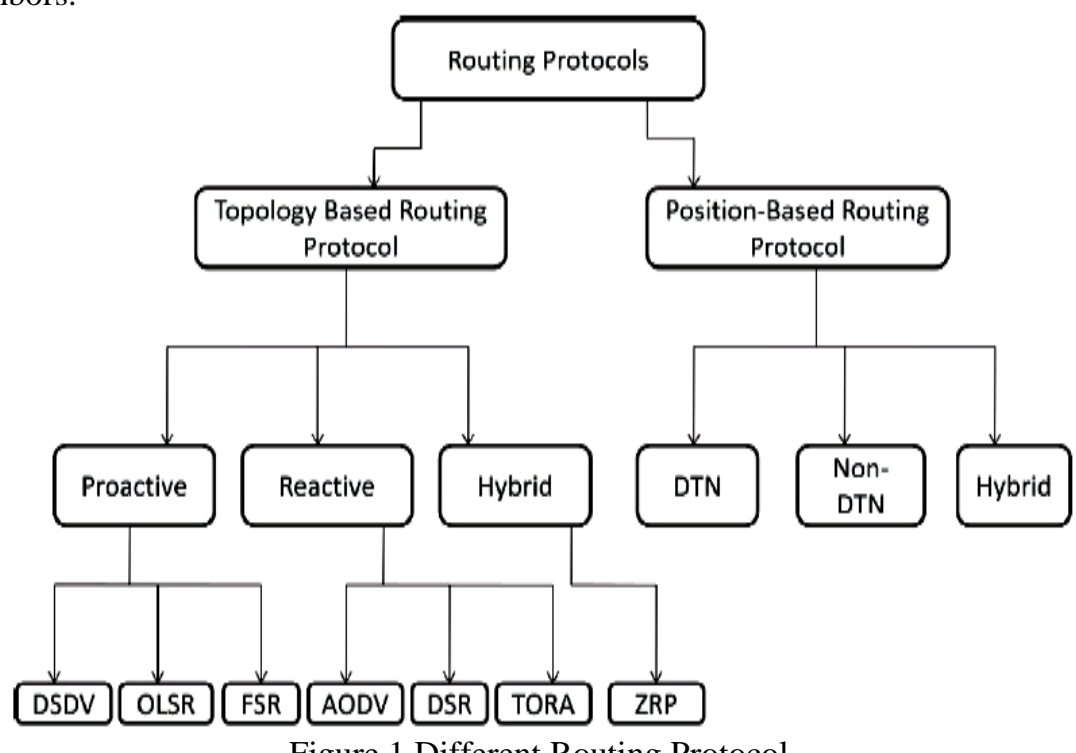

Figure 1 Different Routing Protocol

Every hub finds out about others adjacent and how to contact them, and may report that it also can contact them. Mobile Ad Hoc Networks (MANETs) are application of Mobile Ad-Hoc Network (MANETs). MANETs were first 
said and introduced in 2001 under "auto to-auto uniquely named compact correspondence and systems administration" applications, where systems can be surrounded and information can be given off among cars. It was demonstrated that vehicle-to-vehicle and vehicle-to-roadside trades structures will exist together in MANETs to give road security, course, and other roadside administrations. MANETs are a key bit of the keen transportation systems structure. A portion of the time, MANETs are evaded as Keen Transportation Networks. By 2015, the term MANET wound up being commonly synonymous with the more non specific term between vehicle correspondence (IVC), despite the manner in which that the consideration stays regarding unconstrained systems organization, widely less on the use of structure like Road Side Units (RSUs) or cell systems.

The system is impromptu since it doesn't depend upon a prior structure, for example, switches in wired systems or passages in administered (foundation) remote systems. Or on the other hand perhaps, every middle values controlling by sending information for different focuses, so the confirmation of which focus focuses forward information is made progressively in perspective on brains availability and the organizing figuring being utilized. In the Windows working structure, without any preparation is a correspondence mode (setting) that engages PCs to direct converse with one another without a switch. Dynamic systems in which focuses are allowed to move. VANETs bolster a wide scope of applications - from straightforward one jump information dissemination of, e.g., helpful mindfulness messages (CAMs) to multi-bounce dissemination of messages over immense separations. The vast majority of the concerns important to mobile ad hoc networks (MANETs) are of enthusiasm for VANETs, however the subtleties differ.[5] Rather than moving aimlessly, vehicles will in general move in a composed fashion. The interactions with roadside gear can similarly be described decently precisely. Lastly, most vehicles are limited in their scope of motion, for instance by being constrained to pursue a cleared interstate.

- $\quad$ Electronic brake lights, which permit a driver (or an autonomous vehicle or truck) to respond to vehicles braking despite the fact that they may be clouded (e.g., by other vehicles).

- Platooning, which enables vehicles to firmly (down to a couple of inches) pursue a leading vehicle by remotely getting acceleration and controlling information, in this way shaping electronically coupled "road trains".

- Traffic information systems, which use VANET communication to give up-to-the moment obstruction reports to a vehicle's satellite navigation framework

- $\quad$ Road Transportation Crisis Services - where VANET communications, VANET networks, and road wellbeing cautioning and status information dissemination are utilized to decrease postponements and accelerate crisis salvage operations to spare the lives of those harmed.

- On-The-Road Services - it is likewise envisioned that the future transportation thruway would be "informationdriven" or "remotely empowered". VANETs can help advertise services (shops, corner stores, eateries, and so forth.) to the driver, and even send notifications of any deal going on moment.

\section{LITERATURE SURVEY}

Harinder Kaur, Meenakshi, Vehicular Ad hoc network (VANET) is a heightening field of research and laid reason for some more current innovations like Intelligent Transport Systems (ITS). Routing in VANETs assumes vital job in execution of networks. VANET protocols are named topology based and position based protocols. Research demonstrated that position based protocols are progressively fit to VANETs when contrasted with topology based protocols in light of the fact that geographic routing does not include an overhead and postponement of keeping up routing tables instead geographic position of hubs is utilized for routing which can be gotten by Worldwide Positioning Framework (GPS) gadget on vehicles. In this paper, two geographic routing protocols Grapple based Road and Traffic Mindful Routing (A-STAR) and Insatiable Edge Stateless Routing (GPSR) protocols are assessed on genuine city map. Simulation of VANETs on genuine guide situations give exact outcomes and furthermore helpful to plan and send VANETs in genuine world. True portability model is significant in light of the fact that it reflects true execution of protocols considered. Examination of execution is conveyed as far as throughput, parcel conveyance proportion, bundle misfortune and normal deferral. Simulation of protocols is conveyed by shifting thickness of hubs. A-STAR demonstrated better execution on genuine city map over GPSR on the grounds that A-STAR adopted Road mindfulness strategy for routing though GPSR chips away at Ravenous sending and Routing around the edge methods.[1]

W. Farooq, et.al, The Independent Ground Vehicles (AGVs) are created to play out the secure exercises autonomously to give prosperity to human lives, for instance, in mines identification and breathing space undertakings. The execution of these AGVs has been upgraded in our past work by realizing the Mobile Off the cuff Framework (MANET) among these vehicles. In this bit of research, the Independent Airborne and Ground Vehicles (AAGVs) directing convention has been proposed, in which the aeronautical vehicles are familiar with crushed the confinements of AGVs correspondence for dispersing the Mines Disclosure Messages (MDMs). In addition, the bundle based arrangement is delineated to such an extent that the AAGVs convention can adjust continuously without affecting its execution by keeping up stable Between Mobile Correspondence (IVC) joins. The reenactment of the proposed convention in 
Framework Test framework depicts that the postponement and overhead have been reduced. Then once more, the bundle conveyance proportion and throughput have been extended by grasping the multicast correspondence approach. [2]

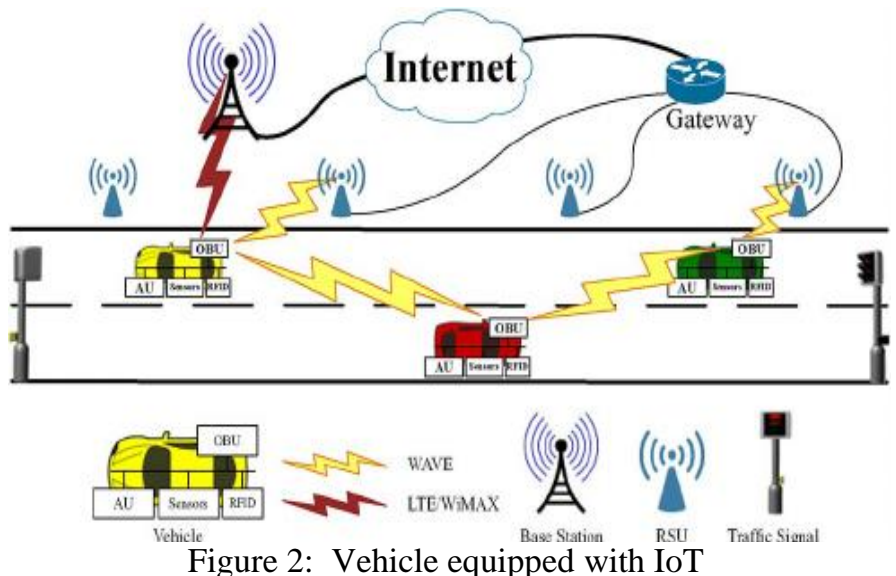

W. Farooq, M. A. Khan, Unmanned Military Vehicles (UMVs) and free robots ended up being a bit of present day battling technique to perform military fight missions and hazardous war field exercises. The military vehicles (MVs) need to talk with one another to achieve a couple of required military endeavors all things considered. It has been cultivated by proposing a self-decision military vehicles coordinating (AMVR) convention to develop a Mobile extraordinarily designated framework (MANET) among all military watched out for and unmanned vehicles to address the challenges of current battling. AMVR convention performs multicast correspondence among unmanned and watched out for military vehicles in mix to make strong coordination among them. The proposed convention plays out the message spread among MVs in two dimension structure for example T1 and T2 which reduces the framework overhead by conveying it among the two dimensions. The UMVs are amassed in to T1 in light of the way that these vehicles can sort out them at front independently with uniform separation by sharing speed and course which avoids the occasion of framework discontinuity also. From now on, the UMVs keep up the steady radio connections of MANET inside one of a kind condition of war field. The occasion revelation messages (EDMs) are spread from unmanned vehicles to watched out for military vehicles (MMVs) of T2. The proposed convention performs multicast correspondence to achieve high throughput and profitable dispersal of EDMs among all or specific gathering of military vehicles. The store and convey approach is grasped to teach moving toward MVs about the current situation of war field. The reenactment comes about speak to that the proposed convention has achieved the target of EDMs dispersal among all UMVs and MMVs successfully regardless of dynamic front line condition. [3]

Z. He, D. Zhang et.al, An item portrayed compose empowers the creation of an adaptable framework configuration by abstracting stream control from solitary gadgets to the framework level. In this paper, we address the troubles in applying SDN to develop unrivaled Mobile systems. We show SDVN, another SDN based Mobile framework building. It makes the topology out of the Mobile systems and utilizations vehicle direction expectation to mitigate the overhead of the SDN control and information plane correspondence. In addition, we propose a multicast convention over SDVN, as multicast is the foundation of various Mobile framework applications. The convention mishandles the framework topology information gave by SDVN to settle on unquestionably gainful multicast arranging decision. The multicast booking issue is intended to restrict the correspondence cost with constrained concede prerequisite. A polynomial time gauge calculation is proposed. We lead broad preliminaries using movement pursues. The appraisal demonstrates that the SDVN based multicast convention beats existing decentralized procedures. [4]

I. Rashdan, Dynamic course masterminding is one of the ITS efficiency applications that decrease travel time and essentialness utilization. To perform capable course orchestrating, constant action information should be gathered and dissipated to vehicles on other road sections. Mobile uniquely designated systems (MANETs) are a promising innovation that can give the correspondence expects to convey the required action information. In this paper, we ponder and survey three bleeding edge dispersing conventions in reasonable Mobile situations. The surveyed conventions are geocast-based reference point piggybacking convention, a low-overhead adjustment of it and enthusiastic edge stateless guiding (GPSR). As execution estimations we measure the ordinary start to finish delay, typical coordinating overhead and the group conveyance proportion as a component of development thickness. Reproduction comes about demonstrate that all of the three conventions achieve the requirements of dynamic course organizing in start to finish postpone and package conveyance proportion. In any case, an enormous diminishment in the coordinating overhead can be cultivated by the changed piggybacking convention. [5] 
The following is a list of some ad hoc network routing protocols.

- Table-driven (proactive) routing

- Respective amount of data for maintenance.

- On-demand (reactive) routing

- High latency time in route finding.

- $\quad$ ABR - Associativity-Based Routing

- Ad hoc On-demand Distance Vector(AODV)

- Dynamic Source Routing

- Flow State in the Dynamic Source Routing

- Power-Aware DSR-based

- Hybrid (both proactive and reactive) routing

- ZRP (Zone Routing Protocol)

- $\quad$ ZHLS (Zone-based Hierarchical Link State Routing Protocol)

- On demand multiple routing protocol (ODMRP)

A. Hierarchical routing protocols

With this kind of protocol the decision of proactive and of receptive routing relies upon the hierarchic dimension wherein a hub resides. The routing is at first settled with some proactively prospected courses and after that serves the interest from additionally initiated hubs through receptive flooding on the lower levels. The decision for one or the other technique requires appropriate attributation for particular dimensions. The fundamental disadvantages of such calculations are:

- $\quad$ CBRP (Cluster Based Routing Protocol)

- $\quad$ FSR (Fisheye State Routing protocol)

- Order One Network Protocol

- $\quad$ ZHLS (Zone-based Hierarchical Link State Routing Protocol)

The Vehicular Receptive Routing (VRR) protocol is incorporated with the WAVE stack and is inserted at the Rationale Connection Control layer. VRR is a multi-station protocol which activities proficient course revelation, course upkeep and information convey forms with the utilization of the Control Channel $(\mathrm{CCH})$ and an Administration Channel (SCH). Standard WSA messages are transmitted over $\mathrm{CCH}$ and IPv6 parcels are transmitted over SCH. To acquire a present neighbor location the WSA edge is changed to convey position information (brings about an additional 4 Bytes). Course solicitation and course answer requests are transmitted inside WSA outlines (additional 40 Bytes) over the Control Channel and information affirmation and all application information (IPv6 parcels) are sent over the Administration Channel. Because of these modifications, a course is right off the bat set up over the $\mathrm{CCH}$ and a while later information is transmitted over the $\mathrm{SCH}$.

Table-I: Comparison of some Protocols

\begin{tabular}{|l|l|l|}
\hline AUTHOR & PROTOCOL & PERFORMANCE \\
\hline Hafez Seliem & MAC \& VDNET & $60 \mathrm{~S}$ \\
\hline Jos ${ }^{\text {E }}$ Grimaldo & AODV, OLSR, DSR, And DSDV & 1 S To $300 \mathrm{~S}$ \\
\hline Forough Goudarzi & Routing Protocol & $800 \mathrm{~S}$ \\
\hline Bhuvaneswari Madasamy & MGOR & $100 \mathrm{~s}$ \\
\hline Guiyang Luo & SDNMAC & $300 \mathrm{~s}$ \\
\hline
\end{tabular}

\section{CONCLUSION}

In this paper, we have explored the distinctive routing protocols for between vehicle communications in VANET. By examining diverse routing protocol in VANET we have seen that further presentation evaluation is required to check execution of a routing protocol with other routing protocols dependent on different traffic situations. Presently multi day's ODMRP protocol is utilizing generally in numerous application. Therefore in protocol based VANET design, can be utilized such protocol. 


\section{REFERENCES}

[1]. Harinder Kaur, Meenakshi, “Analysis of VANET Geographic Routing Protocols on Real City Map” IEEE International Conference On Recent Trends in Electronics Information \& Communication Technology (RTEICT), May 19-20, 2017, India

[2]. W. Farooq, M. A. Khan, S. Rehman, N. A. Saqib and M. Abbas, "AAGV: A Bunch Based Multicast Steering Convention for Self-governing Ethereal and Ground Vehicles Correspondence in VANET," 2017 Global Gathering on Wildernesses of Data Innovation (FIT), Islamabad, 2017, pp. 315-320.

[3]. W. Farooq, M. A. Khan and S. Rehman, "AMVR: A multicast steering convention for self-sufficient military vehicles correspondence in VANET," 2017 fourteenth Worldwide Bhurban Meeting on Connected Sciences and Innovation (IBCAST), Islamabad, 2017, pp. 699-706.

[4]. Z. He, D. Zhang, S. Zhu, J. Cao and X. Liu, "SDN Empowered Superior Multicast in Vehicular Systems," 2016 IEEE 84th Vehicular Innovation Gathering (VTC-Fall), Montreal, QC, 2016, pp. 1-5.

[5]. I. Rashdan, F. de Ponte Muller and S. Sand, "Execution Assessment of Activity Data Dispersal Conventions for Dynamic Course Arranging Application in VANETs," 2016 IEEE 84th Vehicular Innovation Meeting (VTC-Fall), Montreal, QC, 2016, pp. 1-5.

[6]. A. T. Reza, T. A. Kumar and T. Sivakumar, "Position Expectation based Multicast Directing (PPMR) utilizing Kalman Channel over VANET," 2016 IEEE Worldwide Meeting on Building and Innovation (ICETECH), Coimbatore, 2016, pp. 198-206.

[7]. W. Farooq, M. A. Khan and S. Rehman, "A group based multicast directing convention for Self-sufficient Unmanned Military Vehicles (AUMVs) correspondence in VANET," 2016 Universal Meeting on Figuring, Electronic and Electrical Designing (ICE 3D square), Quetta, 2016, pp. 42-48.

[8]. A. Singh and N. Kaur, "Improved Data transmission Proficient Bunch Based Multicasting Convention in VANETs," 2015 second Universal Gathering on Late Advances in Designing and Computational Sciences (RAECS), Chandigarh, 2015, pp. 1-6.

[9]. I. B. Jemaa, O. Shagdar, F. J. Martinez, P. Garrido and F. Nashashibi, "Expanded portability administration and steering conventions for web to-VANET multicasting," 2015 twelfth Yearly IEEE Buyer Correspondences and Systems administration Gathering (CCNC), Las Vegas, NV, 2015, pp. 904-909.

[10]. A. Joshi and R. Kaur, "A novel multi-cast steering convention for VANET," 2015 IEEE Global Propel Figuring Meeting (IACC), Banglore, 2015, pp. 41-45.

[11]. D. Perdana, M. Nanda, R. Tribute and R. F. Sari, "Execution assessment of Panther steering convention for Manhattan portability show on vehicular specially appointed system," 2015 22nd Worldwide Gathering on Broadcast communications (ICT), Sydney, NSW, 2015, pp. 80-84. 